\title{
PD-L1 expression on routine samples of non-small cell lung cancer: results and critical issues from a 1-year experience of a centralised laboratory
}

\author{
Elena Vigliar, Umberto Malapelle, ${ }^{\circledR}$ Antonino laccarino, Gennaro Acanfora, \\ Pasquale Pisapia, Eduardo Clery, Caterina De Luca, Claudio Bellevicine, \\ Giancarlo Troncone
}

Department of Public Health, University of Naples 'Federico II', Naples, Italy

\section{Correspondence to} Professor Giancarlo Troncone, Department of Public Health, University of Naples Federico II, Naples 5 I-80131, Italy; giancarlo.troncone@unina.it

Received 23 January 2019 Revised 13 February 2019 Accepted 14 February 2019 Published Online First 7 March 2019

\section{Check for updates}

(C) Author(s) (or their employer(s)) 2019. No commercial re-use. See rights and permissions. Published by BMJ.

To cite: Vigliar $E$,

Malapelle U, laccarino A,

et al. J Clin Pathol

2019:72:412-417.

\begin{abstract}
Aims Our laboratory is a centralised centre receiving routine non-small cell lung cancer (NSCLC) samples for programmed death ligand-1 (PD-L1) immunohistochemical (IHC) evaluation. Since literature data are not concordant here we review our clinical records to assess the rate of PD-L1 positive and negative NSCLC cases in real-world practice.
\end{abstract}

Methods PD-L1 expression was evaluated by a validated 22C3 IHC laboratory developed test on 211 prospectively collected routine NSCLC samples, received from 10 outside institutions. PD-L1 expression was assessed by the tumour proportion score (TPS) and reported by using a three cut-point system: TPS $<1$, TPS $1 \%-49 \%$ and TPS $\geq 50 \%$.

Results Overall, 193 out of 211 samples (91.5\%) meet the criteria for adequacy (more than 100 viable neoplastic cells). In 62.7\% (121/193) of samples TPS was $<1 \%$; $17.6 \%$ of samples (34/193) expressed PD-L1 with a TPS of $1 \%-49 \%$ and $19.7 \%(38 / 193)$ with a TPS of $>50 \%$. There was no significant difference in PD-L1 expression rates between different histotypes and site of sampling. Instead, a statistically significant difference was associated to the type of samples: in fact, cytological samples were more frequently negative for PD-L1 expression (TPS $<1 \%$ ) and less often displayed PD-L1 expression at high levels (TPS $>50 \%$ ) than surgical resections and biopsies.

Conclusions We present a referral laboratory experience on IHC PD-L1 expression of prospectively collected routine NSCLC samples. Data from the realworld practice can better clarify the percentage of PD-L1 positive and negative cases, to establish benchmarks for good practice standards.

\section{INTRODUCTION}

Immunotherapy, and in particular antibodies $(\mathrm{Ab})$ targeting programmed death 1 (PD-1)/programmed death ligand-1 (PD-L1), is a relevant clinical option in advanced non-small cell lung cancer (NSCLC) management. ${ }^{1}$ However, the immune-checkpoint inhibitors efficacy is limited to a subset of patients and for this reason, the identification of predictive biomarkers is pivotal. In this setting, PD-L1 immunohistochemical (IHC) expression is a predictive biomarker of response to immunotherapy. In particular, the association between PD-L1 expression and treatment by pembrolizumab, a monoclonal human
IgG4 antibody against human PD-1, has been largely investigated. ${ }^{23}$

As a general rule, higher levels of PD-L1 expression are associated to increasing treatment benefit. ${ }^{4}$ PD-L1 expression is scored assessing the percentage of positive neoplastic cells (tumour proportion score, TPS). Positive cells are recognised by a complete or incomplete linear membrane pattern of staining; several studies have shown that in tumours with high TPS, pembrolizumab may be more efficient than chemotherapy, both in terms of progression-free survival and in overall survival (OS). ${ }^{56}$

In particular, pembrolizumab can be considered as a new standard first-line treatment, according to the phase III KEYNOTE-024 trial, carried out in patients with advanced NSCLC with high PD-L1 expression, defined as expression in at least $50 \%$ of tumour cells (TPS $\geq 50 \%$ ), and absence of EGFR or $A L K$ aberrations. ${ }^{6}$ Of interest, in the clinical trial KEYNOTE-042, pembrolizumab improved OS respect to carboplatin-based chemotherapy even in patients with low PD-L1 expression (TPS $\geq 1 \%$ ). ${ }^{5}$

Beyond pembrolizumab, also other drugs that target the PD-1/PD-L1 immunocheckpoint such as the anti-PD-1 nivolumab, and the anti-PD-L1 atezolizumab and durvalumab are available, each linked to a specific anti-PD-L1 Ab clones. In particular, the use of 22C3 clone (Dako, Carpinteria, CA, USA) was associated to inform on pembrolizumab administration; the clones 28-8 (Dako), SP142 (Ventana, Tucson, AZ, USA) and SP263 (Ventana) were associated to nivolumab, atezolizumab and durvalumab treatments, respectively. ${ }^{7}$

Interestingly, these clones have been differently classified by the Food and Drug Administration (FDA). While this latter recognised that there is an undeniable clinical correlation between the 22C3 pharmDx (Dako) and pembrolizumab ('companion diagnostic' assay), ${ }^{8}$ the PD-L1 IHC 28-8 pharmDx (Dako) and PD-L1 SP142 assay (Ventana) were approved simply as 'complementary diagnostic' assays to help oncologists' decisions on nivolumab and atezolizumab administration. To make possible the implementation of PD-L1 testing in clinical practice, the 'chaos' generated by the plethora of different clones and IHC assays needs to be cleared. To this end, several harmonisation studies were carried out to assess the concordancy rate among the different assays. In particular, the Blueprint project showed that three different clones (22C3, 
28-8 and SP263) yield a similar percentage of PD-L1 positive tumour cells. Conversely, a fourth clone (SP142) consistently labelled less neoplastic cells. ${ }^{7}$ Based on this evidence, the 22C3, SP263 and 28-8 clones are currently interchangeably employed by pathologists on different immunostaining platforms (Dako Autostainer Link 48, Dako Omnis, Leica Bond-III, Ventana Bench-Mark ULTRA). Besides FDA-approved assays, laboratory developed test (LDT) can also be used provided a careful analytical validation.

Beyond analytical variables linked to IHC procedures, a further level of complexity is related both to scoring reproducibility and the adoption of more than one cut-off level (1\%, 5\%, 10\%, 25\% and $50 \%$ ) to qualify a single case positive for PD-L1 IHC. ${ }^{9}$ Last but not least, tissue sample type also matters. Resection specimen is not always available in patients with advanced stage NSCLC and small histological biopsies or cytological samples may be the only sources for both morphological and molecular characterisation. PD-L1 IHC procedures have been only validated on formalin-fixed paraffin-embedded (FFPE) samples ${ }^{10} 11$ while immunostaining on smeared material is often unfeasible, thus a cell block (CB) should be prepared to make cytological samples testable.

Taking into account all these points is not surprising that to address clone harmonisation, analytical validation and scoring reproducibility issues, a growing body of literature is being assembled on PD-L1 assays. ${ }^{72-27}$ However, most data have been generated from selected patients that have available satisfactory FFPE tissue specimens. Conversely, in clinical practice samples selection is unfeasible. Thus, data from the real-world practice can better clarify the percentage of PD-L1 positive and negative cases, to establish benchmarks for good practice standards. ${ }^{28} 29$

The molecular laboratory at the pathology department of the University of Naples 'Federico II' is a large-volume centralised laboratory for solid tumour molecular testing for Southern Italy. Since 2017, we have been performing a PD-L1 IHC and here we review our clinical records discussing the differences and similarities with previous literature.

\section{MATERIALS AND METHODS Clinical samples}

From January 2017 to April 2018 we received 211 consecutive oncologists' requests for IHC PD-L1 evaluation. Our laboratory received samples from 10 outside institutions. On receipt of any sample, available information relative to clinical presentations, pathological diagnosis, sample site and type was recorded.

\section{PD-L1 immunohistochemistry by LDT assay}

Our LDT test consisted of the use of Dako's concentrate 22C3 anti-PD-L1 primary antibody with a Ventana's detection systems on the BenchMark XT platform. The samples were prepared as follows: blocks were sectioned at a thickness of 3 $\mu \mathrm{m}$ and stained on positively charged glass slides. Deparaffinisation by EZ Prep solution, rehydration by Reaction Buffer and antigen retrieval were performed by CC1 $(\mathrm{pH} 8.0)$ antigen retrieval solution (Ventana Medical Systems), performed on the VENTANA BenchMark XT automated slide stainer for $60 \mathrm{~min}$ at $100^{\circ} \mathrm{C}$. Specimens were incubated with primary mouse antiPD-L1 monoclonal antibody using a concentration of 1:25 for $80 \mathrm{~min}$ at $37^{\circ} \mathrm{C}$, followed by visualisation with the UltraView DAB IHC Detection Kit (Ventana) and UltraView Amplification Kit (Ventana). The specimens were then counterstained with haematoxylin I and bluing reagent (Ventana) and coverslipped. Each IHC run contained a positive control (in-house control, on-slide tonsil tissue) and a negative antibody control (no primary antibody).

\section{LDT validation}

As requested for any LDT, before clinical implementation a careful validation process was undertaken. To this end, we compared our LDT assay with the gold standard represented by the companion diagnostic PD-L1 IHC 22C3 pharmDx assay performed on the Dako ASL48 platform, according to the FDA specification. ${ }^{8}$ In particular, $\mathrm{n}=21$ NSCLC samples ( $\mathrm{n}=18$ histological samples and $n=3 C B$ ) were selected to include both negative cases $(<1 \%)$, low and high PD-L1 expressors (1\%-49\%; $>50 \%$ ) considering clinical relevant threshold. Each single case was stained by both procedures; a panel of three pathologists evaluated the concordancy between assays. In particular, each case was considered concordant if the score falls within the same category of TPS.

\section{PD-L1 scoring}

Our testing workflow included the assessment of tumour cell content by fully qualified pathologists (EV, CB, GT). A minimum of 100 cells was required to consider a sample eligible for IHC PD-L1 evaluation. Partial or complete linear membrane staining perceived distinct from cytoplasmic staining was considered for TPS evaluation. Only viable tumour cells were scored. Neoplastic cells showing only cytoplasmic staining and positive immune cells (macrophages and lymphocytes) were not scored. The scoring was reported by using a three cut-point system: no PD-L1 expression (TPS $<1 \%$ ), low PD-L1 expression (TPS $1 \%-49 \%$ ) and high PD-L1 expression (TPS $\geq 50 \%$ ). These thresholds were chosen considering their clinical relevance. In fact, patients with more than 50\% TPS can be treated in first line with pembrolizumab, whereas patients with an expression ranging from $1 \%$ to $49 \%$ can be eligible for second-line immunotreatment. Any single case was evaluated jointly at multiviewer microscope by at least three pathologists (EV, CB, GT), especially dedicated to lung pathology and certified to assess PD-L1 22C3 staining by a 2-day educational course (http://www.targosgmbh de/training-courses.html), and any discordance was resolved on consultation.

\section{RESULTS}

\section{LDT validation}

As discussed in the Materials and methods section, a preliminary LDT validation was undertaken comparing our assay to the one approved by FDA as a 'companion diagnostic' (PD-L1 IHC 22C3 pharmDx assay). A 100\% concordance rate was observed when cases were assigned to the three clinical relevant categories. In fact, both assays classified 11 cases as negative (TPS $<1 \%$ ); in addition, six cases were classified as low expressors (TPS $1 \%-49 \%$ ) and four cases as high expressors (TPS $\geq 50 \%$ ).

\section{Clinical samples}

The series included samples from 147 men and 64 women presenting with advanced NSCLC, with an age range of 20-84 years (median age 65 years); one sample was tested for each patient. Sample types submitted for testing included histological biopsies $(n=112,53.1 \%)$, surgical resections $(n=51,24.2 \%)$ and fine needle aspiration (FNA) biopsy specimens ( $\mathrm{n}=48,22.7 \%)$. Sample preparations included paraffin blocks $(\mathrm{n}=129,61.1 \%)$, unstained paraffin sections ( $n=34,16.1 \%), C B s(n=45,21.3 \%)$, direct smears $(n=2,0.9 \%)$ and a liquid-based cytology vial $(\mathrm{n}=1,0.5 \%)$. Submitted material was obtained from primary 


\begin{tabular}{|c|c|}
\hline Characteristic & Received n (\%) \\
\hline \multicolumn{2}{|l|}{ Gender } \\
\hline Male & $147(69.7)$ \\
\hline Female & $64(30.3)$ \\
\hline \multicolumn{2}{|l|}{ Sample type } \\
\hline Biopsy & $112(53.1)$ \\
\hline Surgical resection & $51(24.2)$ \\
\hline FNA & $48(22.7)$ \\
\hline \multicolumn{2}{|l|}{ Sample preparation } \\
\hline Paraffin blocks & $129(61.1)$ \\
\hline Unstained paraffin section & $34(16.1)$ \\
\hline Cell block & $45(21.3)$ \\
\hline Direct smear & $2(0.9)$ \\
\hline Liquid-based cytology vial & $1(0.5)$ \\
\hline \multicolumn{2}{|l|}{ Sample site } \\
\hline Lung & $95(45.0)$ \\
\hline Metastasis & $51(24.2)$ \\
\hline NR & $65(30.8)$ \\
\hline \multicolumn{2}{|l|}{ Diagnosis } \\
\hline$A D C$ & $104(49.3)$ \\
\hline SQCC & $36(17.1)$ \\
\hline NSCLC NOS & $28(13.3)$ \\
\hline Carcinosarcoma & $2(0.9)$ \\
\hline Adenosquamous carcinoma & $1(0.5)$ \\
\hline $\mathrm{SCC}$ & $1(0.5)$ \\
\hline NR & $39(18.5)$ \\
\hline
\end{tabular}

$A D C$, adenocarcinoma; $F N A$, fine needle aspiration; $I H C$, immunohistochemical; NOS, not otherwise specified; NR, not reported;NSCLC, non-squamous cell lung cancer;; PD-L1, programmed death ligand-1; SCC, small cell carcinoma; SQCC, squamous cell carcinoma.

lung tumours $(n=95,45.0 \%)$ or metastases $(n=51,24.2 \%)$; in $65(30.8 \%)$ cases sample site had not been reported. Overall, pathological reports were available in $n=172(81.52 \%)$ cases including a range of different diagnosis; most cases were either adenocarcinoma (ADC) $(n=104,49.3 \%)$ or squamous cell carcinoma $(n=36,17.1 \%)$. NSCLC not otherwise specified was tested in $\mathrm{n}=28$ instances $(13.3 \%)$; less frequent diagnosis included carcinosarcoma $(n=2)$, adenosquamous carcinoma $(n=1)$ and small cell carcinoma $(n=1)$. Data are summarised in table 1 .

\section{PD-L1 immunohistochemistry and scoring}

Overall, 193 out of 211 samples (91.5\%) were immunostained by PD-L1 meeting the criteria for adequacy (more than 100 viable neoplastic cells). Inadequate samples included 11 histological and seven cytological samples. More in detail, inadequate samples were represented by $n=9$ paraffin blocks, $n=2$ unstained paraffin sections from biopsies, $n=4 C B, n=2$ direct smears and $n=1$ liquid-based cytology vial.

In 121 out of $193(62.7 \%)$ specimens neoplastic cells lacked staining or showed scattered occasional stained cells below $1 \%$; 34 of $193(17.6 \%)$ expressed PD-L1 with a TPS between 1\% and $49 \%$, and 38 of 193 (19.7\%) expressed PD-L1 with a TPS of $>50 \%$ (table 2 ).

There was no significant difference in PD-L1 expression rates between different histotypes; similarly, when considering the site of sampling, a similar percentage was found in primary and metastatic lesions. Note that since only one sample was tested for each patient, it was no possible to assess differences of staining
Table 2 PD-L1 expression at clinical relevant cut-off of 193 adequate cases

\begin{tabular}{lr}
\hline Tumour proportion score (TPS) & $\mathbf{n}(\%)$ \\
\hline$<1 \%$ & $121(62.7)$ \\
$1 \%-49 \%$ & $34(17.6)$ \\
$>50 \%$ & $38(19.7)$ \\
\hline
\end{tabular}

PD-L1, programmed death ligand-1.

in a single tumour between primary and metastatic sites and to inform on tumour heterogeneity. Data are detailed reported in table 3 .

A statistically significant difference was instead associated to the type of samples. As a general rule, cytological samples were more frequently negative for PD-L1 expression than surgical resections and biopsy. In fact, the rate of negative cases was $80.5 \%$ in FNA $(n=33), 60.8 \%$ in surgical resection $(n=31)$, $55.4 \%$ in biopsy $(n=56)(p<0.05)$. Similarly, FNAs less often displayed PD-L1 expression at high levels (TPS $>50 \%$ ); in fact, the percentage of FNAs expressing PD-L1 more than 50\% was 4.9\%; conversely, histological biopsies showed strong staining $(>50 \%)$ in a significant larger number of cases $(n=29$ [28.7\%]) $(\mathrm{p}<0.05)$.

As far as the preparation type is concerned, a different distribution of PD-L1 staining was also observed. In particular, a significant difference was found between paraffin blocks and unstained paraffin section versus CB at cut-off TPS $<1 \%$ (paraffin blocks $=69 / 120$ [57.5\%], unstained paraffin section $=18 / 32$ [56.3\%], $\mathrm{CB}=33 / 41$ [80.5\%], $\mathrm{p}<0.05)$ and at cut-off TPS $>50 \%$ (paraffin blocks $=29$ [24.2\%], unstained paraffin section $=8$ [25\%], $\mathrm{CB}=2$ [4.9\%], $\mathrm{p}<0.05$ ) (table 3).

\section{DISCUSSION}

The assessment of PD-L1 expression has become part of the standard of care in the management of patients with advanced NSCLC and PD-L1 IHC testing is currently the only available test to assess whether patients with lung cancer are eligible for pembrolizumab as first-line or second-line treatment. In this study, we reported a series of 211 routine clinical samples consecutively received by our laboratory and tested for PD-L1. Our data showed the overall inadequate sample rate is such as low as $8.5 \%$. This is remarkable, since, conversely, in the clinical trial setting, our cases were unselected. Our data also confirmed the feasibility of cytological material for PD-L1 IHC assay in terms of adequacy. ${ }^{20-22}$ In fact, despite a slight higher inadequacy rate $(14.58 \%)$ of cytological samples compared with histological samples $(6.75 \%)$, the difference was not statistically significant $(p=0.08)$. Furthermore, the cytological samples that were not adequate to inform for PD-L1 expression included either direct smears $(n=2)$ or liquid-based cytology vial $(n=1)$. Since IHC assays for PD-L1 have not been developed and validated on 'traditional' cytological preparation in clinical trials, we argue that a $\mathrm{CB}$ should be always prepared when cytology is used to sample advanced NSCLC. Thus, the crucial role of cytopathologist who evaluates on-site the adequacy of aspirated material and triage part of the samples to prepare a $\mathrm{CB}$, it cannot be overemphasised. A resulting advantage lies in the possibility to use $\mathrm{CB}$ also for the evaluation of the overexpression of other relevant biomarkers, such as ALK and ROS $1 .{ }^{30}$ Conversely, PD-L1 testing on direct smear should be performed only in the absence of an FFPE specimen and should be only carried out by laboratories with specific expertise. ${ }^{31-33}$ In fact, there is concern that pitfalls such as non-specific cytoplasmic or heavy background staining 
Table 3 PD-L1 expression in different sample categories

\begin{tabular}{|c|c|c|c|c|c|c|c|c|c|c|c|c|}
\hline \multirow[b]{2}{*}{ TPS } & \multicolumn{4}{|c|}{ Diagnosis, n (\%) } & \multicolumn{2}{|c|}{ Sample site, n (\%) } & \multicolumn{3}{|c|}{ Sample type, n (\%) } & \multicolumn{3}{|c|}{ Sample preparation, $\mathbf{n}(\%)$} \\
\hline & ADC & SQCC & NOS & Other & Lung & Metastasis & FNA & Surgical resection & Biopsy & $\mathrm{CB}$ & Paraffin blocks & $\begin{array}{l}\text { Unstained } \\
\text { section }\end{array}$ \\
\hline$<1 \%$ & $62(63.9)$ & $23(65.7)$ & $14(56)$ & $22(61.1)$ & $53(60.2)$ & $35(70)$ & $33(80.5)$ & $\begin{array}{l}31(60.8) \\
P=0.0412\end{array}$ & $\begin{array}{l}56(55.4) \\
P=0.0052\end{array}$ & $33(80.5)$ & $\begin{array}{l}69(57.5) \\
P=0.0084\end{array}$ & $\begin{array}{l}18(56.3) \\
P=0.0251\end{array}$ \\
\hline $1 \%-49 \%$ & $16(16.5)$ & $8(22.9)$ & $4(16)$ & $6(16.7)$ & $17(19.3)$ & $7(14)$ & $6(14.6)$ & $12(23.5)$ & $16(15.8)$ & $6(14.6)$ & $22(18.3)$ & $6(18.8)$ \\
\hline$>50 \%$ & $19(19.6)$ & $4(11.4)$ & $7(28)$ & $8(22.2)$ & $18(20.5)$ & $8(16)$ & $2(4.9)$ & $8(15.7)$ & $\begin{array}{l}29(28.7) \\
P=0.0018\end{array}$ & $2(4.9)$ & $\begin{array}{l}29(24.2) \\
P=0.0068\end{array}$ & $\begin{array}{l}8(25) \\
P=0.0131\end{array}$ \\
\hline Total & 97 & 35 & 25 & 36 & 88 & 50 & 41 & 51 & 101 & 41 & 120 & 32 \\
\hline
\end{tabular}

Values in bold indicate statistically significant difference in sample type and sample preparation categories between surgical resection/biopsy versus FNA and paraffin blocks/ unstained section versus $\mathrm{CB}$, at cut-off TPS $<1 \%$ and $>50 \%$.

SQCC squamous cell carcinoma; ADC, adenocarcinoma;CB, cell block; FNA, fine needle aspiration; NOS, not otherwise specified; PD-L1, programmed death ligand-1; TPS, tumour proportion score.

and presence of cellular debris may lead to misinterpretation. Thus, laboratories should issue their own guidelines informing oncologists on the procedures that had been validated for PD-L1 testing. Indeed, when our data were reviewed considering direct smears and liquid based cytology (LBC) 'not suitable' rather than 'inadequate', the inadequacy rate of histological samples $(6.75 \%)$ and CBs $(4 / 45 ; 8.8 \%)$ was very similar.

Evaluating the impact of our data on clinical treatment, we report that $62.7 \%$ of patients were considered not eligible for pembrolizumab monotherapy, because of the lack of expression of PD-L1 (TPS $<1 \%)$; the remaining patients were candidates for pembrolizumab therapy as first-line (19.7\%, TPS $>50 \%$ ) or second-line treatment (17.6\%, TPS 1\%-49\%) respectively. Conversely, in the genomic lung cancer predictive biomarkers, whose prevalence in different histotypes has been well established on large series, ${ }^{34}$ the expected percentage of PD-L1 negative and positive cases is not clearly defined; as shown in figure 1, there is a little degree of concordancy on the rate of 22C3 PD-L1 IHC expression. This is evident when considering the rate of negative cases; in clinical trials the percentage of TPS $<1 \%$ was about a

\begin{tabular}{|c|c|c|c|c|c|c|}
\hline & & & & \multicolumn{3}{|c|}{ TPS } \\
\hline Author & Study type & Cases $\left(n^{\circ}\right)$ & Sample type & $<1 \%$ & $1-49 \%$ & $>50 \%$ \\
\hline Garon et al ${ }^{2}$ & clinical trial & 824 & histological & 39.2 & 37.6 & 23.2 \\
\hline Herbst et al ${ }^{3}$ & clinical trial & 2222 & histological & 33.8 & 37.8 & 28.4 \\
\hline Roach et al ${ }^{11}$ & validation & 127 & histological & 57.5 & 23.6 & 18.4 \\
\hline Brunnström et al ${ }^{17}$ & harmonisation & 55 & TMA & 53 & 29 & 18 \\
\hline & & & TMA & 66.8 & 19.5 & 13.7 \\
\hline Hendry et al ${ }^{24}$ & harmonisation & 368 & TMA & 66 & 23.9 & 10.1 \\
\hline Evans et al 28 & routine series & 10005 & histological, cytological & 44.4 & 25 & 30.6 \\
\hline
\end{tabular}

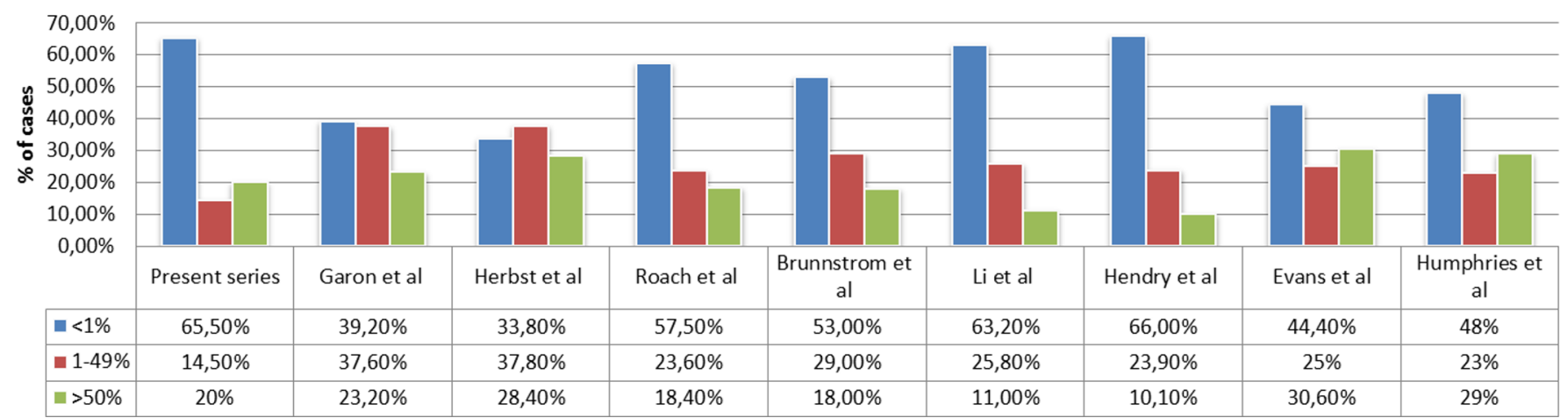

Figure $122 \mathrm{C} 3$ programmed death ligand-1 (PD-L1) immunohistochemical expression rate: literature data and visual comparison. TPS, tumour proportion score. TMA, tissue micro array. 
third of analysed cases, ranging from $33 \%$ to $39 \% .^{23}$ Instead, in validation and harmonisation studies the percentage of TPS $<1 \%$ far more increased, ranging from 53\% to 66\%. ${ }^{11} 161724$ More recently, investigation from routine clinical testing series has shown intermediate values (ranging from 44.4\% to 48\%). ${ }^{28} 29$ Discrepant results are also noticeable for the rates of positive cases. In fact, in clinical trial and routine series TPS $>50 \%$ cases were above 20\% (23\%-30.6\%) whereas in validation and harmonisation study the percentage of TPS $>50 \%$ was below $20 \%$, ranging from $10 \%$ to $18 \%$ (figure 1 ). In our current experience, nearly two-thirds (62.7\%) of analysed samples did not express PD-L1 (TPS $<1 \%$ ), similar to that reported in validation and harmonisation studies (figure 1).

Both technical and clinical issues may explain the difference between our data and those generated by clinical trials. In routine practice, patients are heterogeneous, while clinical trials are conducted in selected populations that meet study inclusion criteria (age, Eastern Cooperative Oncology Group performance status of 0 or 1 , disease progression after first-line chemotherapy and rebiopsy availability). This information is not provided at all to the pathology laboratory, so it is difficult to identify clinical factors that might have had an effect on our data. Likewise, even basic information such as those included in the standard histological reports is often not provided. Since a higher expression of PD-L1 may occur in higher tumour grade and in solid pattern ADC, ${ }^{28}$ and morphomolecular correlation can help understand factors of response to immunotherapy, we recommend primary pathologist to provide more complete information.

Besides clinical considerations, also preanalytical factors may strongly influence PD-L1 staining, both on histological and cytological samples. Recommendations on type and length of fixation (10\% neutral buffered formalin, fixation time between 6 and 48 hours) and cut sections storage modalities (stable temperature for 4-6 weeks) are well suggested ${ }^{35}{ }^{36}$; interestingly, some authors have previously reported a slightly higher rate of high expression of PD-L1 (TPS $>50 \%$ ) in cytology specimens compared with small biopsies or resection specimens. ${ }^{2028}$ As possible explanation relied on the likely probability of delayed or improper overfixation of surgical resection specimens. ${ }^{20}$ This is not confirmed by our results. In fact, in our series, although the overall percentage of non-expressor (TPS $<1 \%$ ) was generally high, the outliner was represented by cytological samples, both considering the cut-off of TPS $<1 \%$ $(80.5 \%)$ and that of TPS $>50 \%(4.9 \%)$. As a matter of fact, it widely held that the lack of standardisation is more common in CB preparation compared with histological samples. In fact, $\mathrm{CB}$ preparatory techniques greatly vary based on the choice of the fluid medium used for the FNA needle rinse (formalin, saline or alcohol-based fixatives followed by formalin after fixation), fixation time and method of concentration, and furthermore on expertise and workload of the laboratory. ${ }^{37}$ Therefore, efforts must be spent to standardise CB handling and processing and further comparative studies are required to understand the influence of CB preparatory techniques (fixatives, fixation time) in PD-L1 IHC results.

In conclusion, in this we present a referral laboratory experience on IHC PD-L1 expression of prospectively collected routine NSCLC samples; the most significant limitations of our study were the low number of cases and the lack of clinical and histological data. Unlike other lung cancer predictive biomarkers, reference ranges for the expected percentage of PD-L1 negative and positive cases are not clearly defined and values of 22C3 PD-L1 IHC results reported in literature until now are not homogeneous. Further investigation is required to evaluate the prevalence of different TPS and to investigate possible factors affecting the PD-L1 positivity distribution. In particular, our experience strongly suggests that updated international guidelines are strongly needed to achieve a better standardisation of PD-L1 testing in clinical practice.

Take home messages

Programmed death ligand-1 (PD-L1) is currently the only predictive biomarker of response to immunotherapy in patients with advanced non-small cell lung cancer.

- Until now, data relative to the rates of PD-L1 expression in clinical routine series are still limited.

- In our practice, cytological samples were more frequently negative for PD-L1 expression and less often displayed PD$\mathrm{L} 1$ expression at high levels than surgical resections and biopsies.

- Updated international guidelines are strongly needed to achieve a better standardisation of PD-L1 testing in clinical practice, in particular regarding cytological preparations.

\section{Handling editor Runjan Chetty.}

Contributors EV, UM and GT conceived the study and wrote the paper. Al and CDL performed all experiments. GA and EC collected the data. CB and PP contributed as pathologists.

Funding The authors have not declared a specific grant for this research from any funding agency in the public, commercial or not-for-profit sectors.

Competing interests None declared.

Patient consent for publication Not required.

Provenance and peer review Not commissioned; internally peer reviewed.

\section{REFERENCES}

1 Mino-Kenudson M, Mino-Kenudson M. Programmed cell death ligand-1 (PD-L1) expression by immunohistochemistry: could it be predictive and/or prognostic in nonsmall cell lung cancer? Cancer Biol Med 2016;13:157-70.

2 Garon EB, Rizvi NA, Hui R, et al. Pembrolizumab for the treatment of NonSmall-Cell lung cancer for the KEYNOTE-001 Investigators*. N Engl J Med 2015;21372:2018-28.

3 Herbst RS, Baas P, Kim D-W, et al. Pembrolizumab versus docetaxel for previously treated, PD-L1-positive, advanced non-small-cell lung cancer (KEYNOTE-010): a randomised controlled trial. Lancet 2016;387:1540-50.

4 Kowanetz M, Zou W, Gettinger SN, et al. Differential regulation of PD-L1 expression by immune and tumor cells in NSCLC and the response to treatment with atezolizumab (anti-PD-L1). Proc Natl Acad Sci U S A 2018;115:E10119-E10126.

5 De Lima Lopes G, Wu Y-L, Sadowski S, et al. P2.43: Pembrolizumab vs Platinum-Based Chemotherapy for PD-L1+ NSCLC: Phase 3, Randomized, Open-Label KEYNOTE-042 (NCT02220894). J Thorac Oncol 2016;11:S244-S245.

6 Reck M, Rodríguez-Abreu D, Robinson AG, et al. Pembrolizumab versus chemotherapy for PD-L1-positive non-small-cell lung cancer. N Engl J Med 2016;375:1823-33.

7 Hirsch FR, McElhinny A, Stanforth D, et al. PD-L1 immunohistochemistry assays for lung cancer: results from phase 1 of the blueprint PD-L1 IHC assay comparison project. J Thorac Oncol 2017:12:208-22.

8 Sul J, Blumenthal GM, Jiang X, et al. FDA approval summary: pembrolizumab for the treatment of patients with metastatic non-small cell lung cancer whose tumors express programmed Death-Ligand 1. Oncologist 2016;21:643-50.

9 Troncone G, Gridelli C. The reproducibility of PD-L1 scoring in lung cancer: can the pathologists do better? Trans/ Lung Cancer Res 2017;6(Suppl 1):S74-S77.

10 Dolled-Filhart M, Roach C, Lubiniecki GM, et al. Emancipator K development of a companion diagnostic for pembrolizumab in Non-Small cell lung cancer using immunohistochemistry for programmed death ligand-1. Arch Pathol Lab Med 2016.

11 Roach C, Zhang N, Corigliano E, et al. Development of a companion diagnostic PD-L1 immunohistochemistry assay for pembrolizumab therapy in non-small-cell lung cancer. Appl Immunohistochem Mol Morphol 2016;24:392-7.

12 Tsao MS, Kerr KM, Kockx M, et al. PD-L1 immunohistochemistry comparability study in real-life clinical samples: results of blueprint phase 2 project. J Thorac Oncol 2018;13:1302-11.

13 Scheel AH, Baenfer G, Baretton G, et al. Interlaboratory concordance of PDL1 immunohistochemistry for non-small-cell lung cancer. Histopathology 2018;72:449-59. 
14 Ilie M, Khambata-Ford S, Copie-Bergman C, et al. Use of the 22C3 anti-PD-L1 antibody to determine PD-L1 expression in multiple automated immunohistochemistry platforms. PLoS One 2017;12:e0183023.

15 Scheel AH, Dietel M, Heukamp LC, et al. Harmonized PD-L1 immunohistochemistry for pulmonary squamous-cell and adenocarcinomas. Mod Pathol 2016;29:1165-72.

16 Li C, Huang C, Mok TS, et al. Comparison of 22C3 PD-L1 expression between surgically resected specimens and paired tissue microarrays in non-small cell lung cancer. J Thorac Oncol 2017;12:1536-43.

17 Brunnström H, Johansson A, Westbom-Fremer S, et al. PD-L1 immunohistochemistry in clinical diagnostics of lung cancer: inter-pathologist variability is higher than assay variability. Mod Pathol 2017;30:1411-21.

18 Cooper WA, Russell PA, Cherian M, et al. Intra- and interobserver reproducibility assessment of PD-L1 biomarker in Non-Small cell lung cancer. Clin Cancer Res;217:4569-77.

19 Rehman JA, Han G, Carvajal-Hausdorf DE, et al. Quantitative and pathologist-read comparison of the heterogeneity of programmed death-ligand 1 (PD-L1) expression in non-small cell lung cancer. Mod Pathol 2017;30:340-9.

20 Wang H, Agulnik J, Kasymjanova G, et al. Cytology cell blocks are suitable for immunohistochemical testing for PD-L1 in lung cancer. Ann Oncol 2018;29:1417-22.

21 Torous VF, Rangachari D, Gallant BP, et al. PD-L1 testing using the clone 22C3 pharmDx kit for selection of patients with non-small cell lung cancer to receive immune checkpoint inhibitor therapy: are cytology cell blocks a viable option? J Am Soc Cytopathol 2018;7:133-41.

22 Heymann JJ, Bulman WA, Swinarski D, et al. PD-L1 expression in non-small cell lung carcinoma: comparison among cytology, small biopsy, and surgical resection specimens. Cancer Cytopathol 2017;125:896-907.

23 Batenchuk C, Albitar M, Zerba K, et al. Burns V. A real-world, comparative study of FDA-approved diagnostic assays PD-L1 IHC 28-8 and 22C3 in lung cancer and other malignancies. J Clin Pathol jclinpath 2018;205362.

24 Hendry S, Byrne DJ, Wright GM, et al. Comparison of four PD-L1 immunohistochemical assays in lung cancer. J Thorac Oncol 2018;13:367-76.

25 Adam J, Le Stang N, Rouquette I, et al. Multicenter harmonization study for PD-L1 IHC testing in non-small-cell lung cancer. Ann Oncol 2018;29:953-8.
26 Marchetti A, Barberis M, Franco R, et al. Multicenter comparison of 22C3 PharmDx (Agilent) and SP263 (Ventana) assays to test PD-L1 expression for NSCLC patients to be treated with immune checkpoint inhibitors. J Thorac Oncol 2017;12:1654-63.

27 Rimm DL, Han G, Taube JM, et al. A prospective, multi-institutional, Pathologist-Based assessment of 4 immunohistochemistry assays for PD-L1 expression in non-small cell lung cancer. JAMA Oncol 2017;3.

28 Evans M, O'Sullivan B, Hughes F, et al. The clinicopathological and molecular associations of PD-L1 expression in non-small cell lung cancer: analysis of a series of 10,005 cases tested with the 22C3 assay. Pathol Oncol Res 2018:1-11.

29 Humphries MP, McQuaid S, Craig SG, et al. Critical appraisal of programmed death ligand 1 reflex diagnostic testing: current standards and future opportunities. J Thorac Oncol 2019;14.

30 Pisapia P, Lozano MD, Vigliar E, et al. ALK and ROS1 testing on lung cancer cytologic samples: perspectives. Cancer Cytopathol 2017;125:817-30.

31 Noll B, Wang W-L, Gong Y, et al. Programmed death ligand 1 testing in non-small cell lung carcinoma cytology cell block and aspirate smear preparations. Cancer Cytopathol 2018;126:342-52.

32 Jain D, Sukumar S, Mohan A, et al. Programmed death-ligand 1 immunoexpression in matched biopsy and liquid-based cytology samples of advanced stage non-small cell lung carcinomas. Cytopathology 2018;29:550-7.

33 Capizzi E, Ricci C, Giunchi F, et al. Validation of the immunohistochemical expression of programmed death ligand 1 (PD-L1) on cytological smears in advanced non small cell lung cancer. Lung Cancer 2018;126:9-14.

34 Ettinger DS, Wood DE, Aisner DL, et al. Non-small cell lung cancer, version 5.2017, NCCN clinical practice guidelines in oncology. J Natl Compr Canc Netw 2017; 15:504-35.

35 Thunnissen E, Kerr KM, Herth FJF, et al. The challenge of NSCLC diagnosis and predictive analysis on small samples. Practical approach of a Working Group. Lung Cancer 2012;76:1-18.

36 Kerr KM, Bubendorf L, Edelman MJ, et al. Second ESMO consensus Conference on lung cancer: pathology and molecular biomarkers for non-small-cell lung cancer. Ann Oncol 2014;25:1681-90.

37 Nambirajan A, Jain D. Cell blocks in cytopathology: an update. Cytopathology 2018;29:505-24 